Draft VERSION NOVEMBER 1, 2018

Preprint typeset using $\mathrm{AT}_{\mathrm{E} X} \mathrm{X}$ style emulateapj v. 08/22/09

\title{
RETAINING BLACK HOLES WITH VERY LARGE RECOIL VELOCITIES
}

\author{
JEREMY D. SCHNITTMAN \\ Department of Physics, University of Maryland, College Park, MD 20742 \\ Draft version November 1, 2018
}

\begin{abstract}
Recent numerical simulations of binary black hole mergers show the possibility of producing very large recoil velocities $\left(>3000 \mathrm{~km} \mathrm{~s}^{-1}\right)$. Kicks of this magnitude should be sufficient to eject the final black hole from virtually any galactic potential. This result has been seen as a potential contradiction with observations of supermassive black holes residing in the centers of most galaxies in the local universe. Using an extremely simplified merger tree model, we show that, even in the limit of very large ejection probability, after a small number of merger generations there should still be an appreciable fraction $(>50 \%)$ of galaxies with supermassive black holes today. We go on to argue that the inclusion of more realistic physics ingredients in the merger model should systematically increase this retention fraction, helping to resolve a potential conflict between theory and observation. Lastly, we develop a more realistic Monte Carlo model to confirm the qualitative arguments and estimate occupation fractions as a function of the central galactic velocity dispersion.
\end{abstract}

Subject headings: black hole physics - galaxies: nuclei

\section{INTRODUCTION}

In the standard model of hierarchical structure formation, the most over-dense regions of the universe collapse at early times to form small gravitationally bound clumps of dark matter and baryons. These proto-galaxies subsequently merge with each other and form larger and larger objects, up to the galaxies and clusters we see today (Press \& Schechter 1974; Efstathiou \& Rees 1988; Haehnelt \& Rees 1993; Cole et al. 2000). Observations of the local universe suggest that most galaxies at redshift $z=0$ are also host to a supermassive black hole (SMBHs) near their centers (Richstone et al. 1998). Observations of distant quasars in the Sloan survey suggest that these SMBHs are common up to redshifts of at least $z \sim 6$ (Fan et al. 2001). One natural explanation is that the SMBHs originally formed in the early universe and then simply followed their host galaxies throughout multiple generations of hierarchical mergers: whenever a pair of galaxies merged, their central black holes would sink together through dynamical friction and eventually merge themselves, resulting in a single, more massive galaxy with a single, more massive $\mathrm{BH}$ at its center (Kauffmann \& Haehnelt 2000; Cattaneo 2001).

In the absence of special symmetries, the final black hole will receive a linear momentum recoil from the gravitational radiation emitted during the binary merger process (Bekenstein 1973; Fitchett 1983). Recent numerical relativity simulations suggest that this recoil cannot exceed $175 \mathrm{~km} \mathrm{~s}^{-1}$ for non-spinning black holes (Herrmann et al. 2006; Baker et al. 2006; Gonzalez et al. 2006), but may be as large as $\sim 3600 \mathrm{~km} \mathrm{~s}^{-1}$ for maximally spinning equal-mass black holes with spins anti-aligned in the orbital plane (Gonzalez et al. 2007; Campanelli et al. 2007). In this latter case, the resulting $\mathrm{BH}$ would almost certainly be ejected from the gravitational well of its host galaxy (Menou et al. 2001; Merritt et al. 2004).

However, since observations of the local universe suggest that SMBHs are quite common, there appears to be something preventing these ejections. As a first attempt at resolving this conflict, Schnittman \& Buonanno (2007) carried out Monte Carlo simulations of the binary $\mathrm{BH}$ recoil for a large range of mass ratios and spin orientations, finding a relatively small fraction $f_{1000} \simeq$ $0.05-0.1$ of systems with kick velocities $\geq 1000 \mathrm{~km} \mathrm{~s}^{-1}$. These fractions correspond to black holes with dimensionless spin parameters of $a / M=0.9$, which appear to be typical from AGN observations (Yu \& Tremaine 2002; Elvis et al. 2002; Wang et al. 2006). More recently, Bogdanovic et al. (2007) proposed a mechanism in which a circumbinary gas accretion disk would align the spins of the two black holes with the orbital axis of the disk prior to merger, thereby limiting the maximum recoil to $\lesssim 200 \mathrm{~km} \mathrm{~s}^{-1}$ (Baker et al. 2007).

In this paper, we take a rather different approach to the problem and question the very premise of the apparent conflict: Does a large recoil velocity necessarily imply a small occupation fraction? Furthermore, does a small occupation fraction at high redshift imply that only a small number of SMBHs will survive until today? If, for example, $95 \%$ of all $\mathrm{BH}$ mergers result in an ejection from the host galaxy, does this really mean that at least $95 \%$ of galaxies today should be devoid of SMBHs? Using a binary tree merger model for the hierarchical growth of $\mathrm{BHs}$ and their host galaxies, we show that the answer to these questions is a resounding "NO!" Our simple analytic predictions appear to agree well with recent work by Volonteri (2007), which employs a much more detailed merger evolution, including $\mathrm{BH}$ evolution due to accretion, but also finds a large occupation fraction at low redshift.

The formal mathematical argument is given below in Section 2, but a simple qualitative reasoning is this: in every generation of the merger tree, the number of galaxies decreases, so the fraction with $\mathrm{BH}$ s can easily increase. If every $\mathrm{BH}$ host galaxy merges with an empty galaxy, then the fraction of galaxies with $\mathrm{SMBHs}$ can double from 0.5 to 1 . Including the losses due to ejected $\mathrm{BHs}$, we find a steady state solution with occupation fraction 
$f=1 /\left(1+p_{\text {ej }}\right)$, where $p_{\text {ej }}$ is the probability that a merging $\mathrm{BH}$ is ejected due to gravitational recoil. In Section 3. we discuss a number of potential physics ingredients that could be added to the simple model, arguing that each one would systematically increase the fraction of observed SMBHs today. In Section 4 we attempt to confirm some of these arguments with a more astrophysically realistic Monte Carlo merger model, and in Section 5 we present our conclusions.

\section{SIMPLE MERGER TREE MODEL}

We begin with the simplest possible model for a galactic merger tree: a binary tree where in each generation every galaxy merges with one "spouse" and produces a single "child" (see Figs. 1 and 2 for a schematic). Each parent galaxy may or may not have a SMBH at its center, and if they both do, then the respective black holes are assumed to merge into a single black hole, which is then ejected due to gravitational recoil with a probability of $p_{\text {ej. }}$ In the zeroth generation, before any mergers, every galaxy is assumed to have a central black hole present. Thus in the first generation, a fraction $f_{1}=1-p_{\text {ej }}$ of galaxies will have SMBHs.

For a galaxy in the $(i+1)^{\text {st }}$ generation, the probability of having a black hole is

$$
\begin{aligned}
f_{i+1}= & 0 \times\left(1-f_{i}\right)^{2} \\
& +f_{i}\left(1-f_{i}\right)+\left(1-f_{i}\right) f_{i} \\
& +\left(1-p_{\mathrm{ej}}\right) f_{i}^{2} \\
= & f_{i}\left(2-f_{i}-p_{\mathrm{ej}} f_{i}\right),
\end{aligned}
$$

where the first line corresponds to having two parents without black holes, the second line represents either a black hole mother or father, and the third line is the probability of both parents having black holes, but the child black hole not getting ejected in the merger. Taking the convergence limit of $f_{i+1}=f_{i}$ for large $i$, we find

$$
f_{\infty}=\frac{1}{1+p_{\mathrm{ej}}} .
$$

This remarkable result suggests that, in the limit of infinite recoil velocity, $50 \%$ of all galaxies will still retain a central black hole. This is possible because, during one generation of galaxy mergers with $f_{i}=0.5$, while effectively half of the black holes are removed by the recoil, the total number of galaxies is also cut in half, thus maintaining a constant fraction of SMBHs.

In Figures 1 and 2 we show examples of the binary merger trees that obey the simple rules described above. The heavy black circles represent black holes, while the empty circles are galaxies without SMBHs. For ejection fractions $p_{\mathrm{ej}}=0.1$ (Fig. 1) and $p_{\mathrm{ej}}=0.9$ (Fig. 2) we show six generations of galaxy mergers, beginning with $f_{1}=1-p_{\text {ej }}$. In both cases, the system approaches the equilibrium fraction $f_{\infty}$ of equation (2) after only a few generations.

For a more quantitative estimate of the "convergence time" that it takes to reach equilibrium, we see from equation (11) that

$$
\begin{aligned}
f_{1} & =1-p_{\mathrm{ej}}, \\
f_{2} & =1-p_{\mathrm{ej}}+p_{\mathrm{ej}}^{2}-p_{\mathrm{ej}}^{3}, \\
f_{3} & =1-p_{\mathrm{ej}}+p_{\mathrm{ej}}^{2}-p_{\mathrm{ej}}^{3}+p_{\mathrm{ej}}^{4}-p_{\mathrm{ej}}^{5}+p_{\mathrm{ej}}^{6}-p_{\mathrm{ej}}^{7}, \\
& \vdots \\
f_{i} & =\sum_{j=0}^{2^{i}-1}(-1)^{j} p_{\mathrm{ej}}^{j} .
\end{aligned}
$$

This series not only converges to equation (2) as expected, but does so at quite a rapid pace. Even for extremely large ejection rates of $p_{\mathrm{ej}}=0.95$ (i.e. $f_{1}=0.05$ ), $f_{i}$ is within $5 \%$ of its asymptotic value of $f_{\infty}=0.513$ after just five generations. Because of this rapid convergence, we find that the final results are independent of the initial occupation fraction $f_{0}$.

\section{ADDITIONAL PHYSICS COMPONENTS}

The model as described above is based on two major simplifying assumptions: a perfect binary merger tree, and a constant ejection probability. Of course, in reality both of these assumptions are in all likelihood invalid. Here we list a number of possible modifications based on more realistic astrophysics, and for each one argue that their inclusion will only increase the fraction of SMBHs observed today.

The binary merger tree assumes that for a given generation, every galaxy has exactly the same mass and merges with exactly one other galaxy, halving the total number of galaxies in each subsequent generation. In practice, most hierarchical merger simulations go backwards in time, using the extended Press-Schechter formalism (Press \& Schechter 1974) to estimate the progenitor masses at a given redshift (Volonteri et al. 2003). Many of these simulations find that a single trunk of the merger tree dominates, with a large number of small branches joining in at different redshifts (Malbon et al. 2006). This means that the mass ratio for a typical (proto-)galaxy merger can be significantly different than unity. Assuming that the SMBH masses scale with their host masses, this suggests that $\mathrm{BH}$ mass ratio will not be unity (Sesana et al. 2007). While the Fitchett scaling for non-spinning black holes favors a mass ratio of about three-to-one for maximum recoil (Fitchett 1983), numerical simulations imply that the supermassive kicks of $\gtrsim 1000 \mathrm{~km} \mathrm{~s}^{-1}$ require nearly-equal masses and spins (Herrmann et al. 2007; Gonzalez et al. 2007; Campanelli et al. 2007; Tichy \& Marronetti 2007), so a wider range of mass ratios will tend to decrease the ejection probability $p_{\text {ej }}$.

Furthermore, as the galaxies evolve along the hierarchical merger tree, their masses will increase, and thus so will their escape velocities. Of course, the BHs are also growing in mass through mergers and accretion during this time, but the recoil problem is strictly scaleinvariant: the kick velocity is a function only of the mass ratio and the dimensionless spin parameters. Thus if the masses of the BHs and their host bulges all double, the recoil will be the same, but the escape velocity will be larger, and thus $p_{\text {ej }}$ will be smaller. In this scenario, even if most of the BHs are ejected at large redshifts, after a 
few generations $f_{i}$ will grow rapidly due to a large number of "single-parent" mergers, ultimately converging to a larger population fraction based on the smaller value of $p_{\text {ej }}$ at late times.

Another effect that may increase the escape velocity is the formation of a triple- $\mathrm{BH}$ system by the relatively prompt merger of three haloes before their respective BHs have time to merge (Hoffman \& Loeb 2007). In many of these cases, one of BHs is ejected by Newtonian three-body interactions, leaving a "normal" binary $\mathrm{BH}$ in a more massive host galaxy (i.e. made of three parents instead of two), further increasing the escape velocity and lowering the ejection probability. However, this same three-body interaction may be strong enough to give both the single and binary a large enough $\mathrm{New}$ tonian recoil so that all three BHs are ejected.

In addition to these modifications to the merger tree physics and escape velocities, there are also a number of processes that more directly affect the actual recoil velocity. Since the largest kicks are found in systems with the BH spins in the orbital plane, any systematic effect that tends to avoid this orientation will thus reduce the expected kick velocity. One particularly strong influence on the spin orientation is the torque produced by a single circumbinary accretion disk, which can align both black hole spins with the orbital angular momentum with high efficiency (Bogdanovic et al. 2007). In this orientation, the maximum recoil should not be more than $\sim 200 \mathrm{~km}$ $\mathrm{s}^{-1}$ (Baker et al. 2007), well below the escape velocities of most present-day galaxies. In the event that there is no surrounding accretion disk (i.e. a "dry merger"), the two black hole spins may become aligned via spin-orbit resonances (Schnittman 2004), but this process likely requires somewhat special initial conditions.

Lastly, there is also the possibility of creating a new $\mathrm{SMBH}$ ex nihilo during the galactic mergers, which typically are accompanied by massive gas inflows to the center of the resulting galaxy Mihos \& Hernquist (1994). This rapid increase in gas density will trigger a burst of massive star formation, which may then proceed to form super-massive stars through runaway mergers (Gurkan et al. 2006), in turn collapsing to form the seeds of SMBHs, which will also be surrounded by copious amounts of fuel to accrete more mass. In this way, orphan black holes can appear in the merger tree, further increasing the overall occupation fraction.

\section{ASTROPHYSICAL MERGER TREE}

In an attempt to verify some of these qualitative claims, we now develop a slightly more physical Monte Carlo merger model, motivated by basic astrophysical arguments and observations of local SMBHs. Instead of using a constant ejection probability and a perfectly binary merger tree of equal-mass BHs and galaxies, we now consider an initially flat $\mathrm{BH}$ mass distribution function $d N / d M_{\bullet} \sim M_{\bullet}^{-1}$, and assume the galaxy mass is proportional to $\mathrm{BH}$ mass. If an occupied galaxy merges with an empty galaxy, we adjust the final BH mass to "agree" with the total mass of both parent galaxies (physically represented by some accretion episode). For an isothermal sphere model, it turns out the galaxy mass is not important in determining the escape velocity, which depends only on the velocity dispersion: $v_{\text {esc }}=2 \sigma_{v}$. The velocity dispersion is in turn deter- mined from the $\mathrm{BH}$ mass via the $M-\sigma$ relation of Ferrarese \& Merritt (2000); Merritt \& Ferrarese (2001):

$$
M_{\bullet} \approx 1.3 \times 10^{8}\left(\frac{\sigma_{v}}{200 \mathrm{~km} \mathrm{~s}^{-1}}\right)^{4.7} M_{\odot} .
$$

In each generation, a fraction $f_{m}$ of all the galaxies (selected randomly) merge. In the limit of $f_{m} \rightarrow 1$ (as in the binary merger tree), the low-mass tail of the distribution is prematurely depleted, and if $f_{m} \rightarrow 0$, no evolution takes place at all. However, we find that for the intermediate range $0.25 \lesssim f_{m} \lesssim 0.75$, the net results are largely independent of $f_{m}$. For each merger, we determine the final occupation as in the binary model: two empty parents create an empty child, a single $\mathrm{BH}$ mother or father will create a $\mathrm{BH}$ child, and in the case of two $\mathrm{BH}$ parents, the child black hole will be ejected if the kick velocity is greater than the escape velocity of the child galaxy.

To determine the kick velocity in this more astrophysically realistic model, we employ the analytic fits presented in Schnittman \& Buonanno (2007), assuming all $\mathrm{BH}$ are rapidly spinning with $a / M=0.9$. In this case, for a given mass ratio $q \equiv m 1 / m 2 \leq 1,90 \%$ of the mergers should produce kick velocities less than $v_{90}$ :

$$
v_{90}(q) \approx \frac{17900 q^{2}}{(1+q)^{5}} \sqrt{(1-q)^{2}+1.4(1+q)^{2}} \mathrm{~km} \mathrm{~s}^{-1},
$$

with the actual recoil $v_{\text {kick }}$ selected randomly from the cumulative distribution function (Schnittman \& Buonanno 2007)

$$
P_{\text {cdf }}\left(v_{\text {kick }} ; q\right)=10^{-v_{\text {kick }}^{2} / v_{90}^{2}(q)} \text {. }
$$

In the upper panel of Figure 3, we plot the occupation fraction as a function of $\sigma_{v}$, employing the model described above for two cases: an initial occupation of $f_{0}=0.99$ (solid curves) and for $f_{0}=0.4$ (dashed curves). In both plots, the fractions $f_{i}$ refer to the average number of mergers for each galaxy, not the number of generations $\left(N_{\text {mergers }} \approx N_{\text {gen }} f_{m} /\left(2-f_{m}\right)\right)$. After a few mergers, both cases converge to the steady-state case of $f_{\infty}$ (heavy black curve), just as in the simple binary merger model. In the lower panel, we repeat the same calculation, now multiplying $v_{90}$ by a factor of three, to see the effect of extremely large kicks. While the occupation fractions clearly decrease somewhat, they remain significantly above $50 \%$, just as predicted by the binary merger model.

We do not claim that this improved model should be seen as reliable for making quantitative astrophysical predictions, but rather present it as an application of the simple binary tree model and a confirmation of many of the qualitative arguments from Section 3 At the same time, it is noteworthy that the occupation fractions for the standard model (upper panel of Fig. 3) seem to agree quite closely with the much more detailed model of Volonteri (2007). We anticipate that future observations should be able to measure this distribution at larger redshifts and smaller $\sigma_{v}$, ultimately explaining how the $\mathrm{BH}$ occupation fraction evolves in time.

\section{CONCLUSIONS}

Based on a very simple binary merger tree model, we find that the fraction of galaxies hosting a SMBH should 
be $\gtrsim 50 \%$ even in the limiting case of very large recoil velocities (or alternatively, very small escape velocities). This would be particularly important at high redshifts, when typical galaxies have small escape velocities and the seeds of today's SMBHs are presumably formed. Including qualitative arguments about the hierarchical growth of the host galaxies, the ejection probability $p_{\text {ej }}$ will tend to decrease with cosmological redshift, further enhancing the fraction of central BHs observed today. Including spin-alignment effects from accretion will increase this fraction even more.

In Section 4, we presented a somewhat more physical
Monte Carlo model and were able both to confirm the predictions of the binary merger tree and also reproduce the basic results of the detailed calculations of Volonteri (2007). We thus conclude that the very large kicks predicted by numerical relativity should not necessarily be seen as contradictory to the seemingly ubiquitous population of galaxies with SMBHs observed today.

The author would like to thank Alessandra Buonanno, Julian Krolik, and Cole Miller for helpful discussions and comments. Partial support comes from NSF grant PHY0603762 .

\section{REFERENCES}

Baker, J. G., Centrella, J., Choi, D.-I., Koppitz, M., van Meter, J. R., \& Miller, M. C. 2006 ApJ, 653, L93.

Baker, J. G., Boggs, W. D., Centrella, J., Kelly, B. J., McWilliams, S. T., Miller, M. C., \& van Meter, J. R. 2007, astro-ph/0702390.

Bekenstein, J. D. 1973, ApJ 183, 657.

Bogdanovic, T., Reynolds, C. S., \& Miller, M. C. 2007, astro-ph/0703054

Campanelli, M., Lousto, C.O., Zlochower, Y. \& Merritt, D. 2007, gr-qc/0701164.

Cattaneo, A. 2001, MNRAS 324, 128.

Cole, S., Lacey, G., Baugh, C. M., \& Frenk, C. S. 2000, MNRAS $319,168$.

Efstathiou, G., \& Rees, M. J. 1988, MNRAS 230, 5.

Elvis, M., Risaliti, G., \& Zamorani, G. 2002, ApJ 565, L75.

Fan, X., et al. 2001, AJ 121, 54.

Ferrarese, L., \& Merritt, D. 2000, ApJ 539, L9.

Fitchett, M. J. 1983, MNRAS 203, 1049.

Gonzalez, J. A., Sperhake, U., Brügmann, B., Hannam, M. D., \& Husa, S. 2006, gr-qc/0610154.

Gonzalez, J. A., Hannam, M. D., M. D., Sperhake, U., Brügmann, B., \& Husa, S. 2007, gr-qc/0702052 .

Gurkan, M. A., Fregeau, J. M., \& Rasio, F. A. 2006, ApJ 640, L39.

Haehnelt, M. G., \& Rees, M. J. 1993, MNRAS 263, 168.
Herrmann, F., Shoemaker, D., \& Laguna, P. 2006, gr-qc/0601026.

Herrmann, F., Hinder, I., Shoemaker, D., Laguna, P., \& Matzner, R. A. 2007, gr-qc/0701143.

Hoffman, L., \& Loeb, A. 2007, MNRAS 377, 957.

Kauffmann, G., \& Haehnelt, M. G. 2000, MNRAS 311, 576.

Malbon, R. K., Baugh, C. M., Frenk, C. S., \& Lacey, C. G. 2006, astro-ph/0607424].

Merritt, D., \& Ferrarese, L. 2001, ApJ 547, 140.

Merritt, D., Milosavljevic, M., Favata, M., \& Hughes, S. A. 2004, ApJ 607, L9.

Mihos, J. C., \& Hernquist, L. 1994, ApJ 437, L47

Menou, K., Haiman, Z., \& Narayanan, V. K. 2001, ApJ 558, 535.

Press, W. H., \& Schechter, P. L. 1974, ApJ 187, 425.

Richstone, D., et al. 1998, Nature 395, A14.

Schnittman, J. D. 2004, Phys. Rev. D, 124020.

Schnittman, J. D., \& Buonanno, A. 2007, ApJ 662, L63.

Sesana, A., Volonteri, M., \& Haardt, F. 2007, astro-ph/0701556.

Tichy, W., \& Marronetti, P. 2007, gr-qc/0703075.

Volonteri, M., Haardt, F., \& Madau, P. 2003, ApJ 582, 559.

Volonteri, M. 2007, astro-ph/0703180.

Wang, J.-M. 2006, ApJ 642, L111.

Yu, Q. J., \& Tremaine, S. 2002, MNRAS 335, 965. 


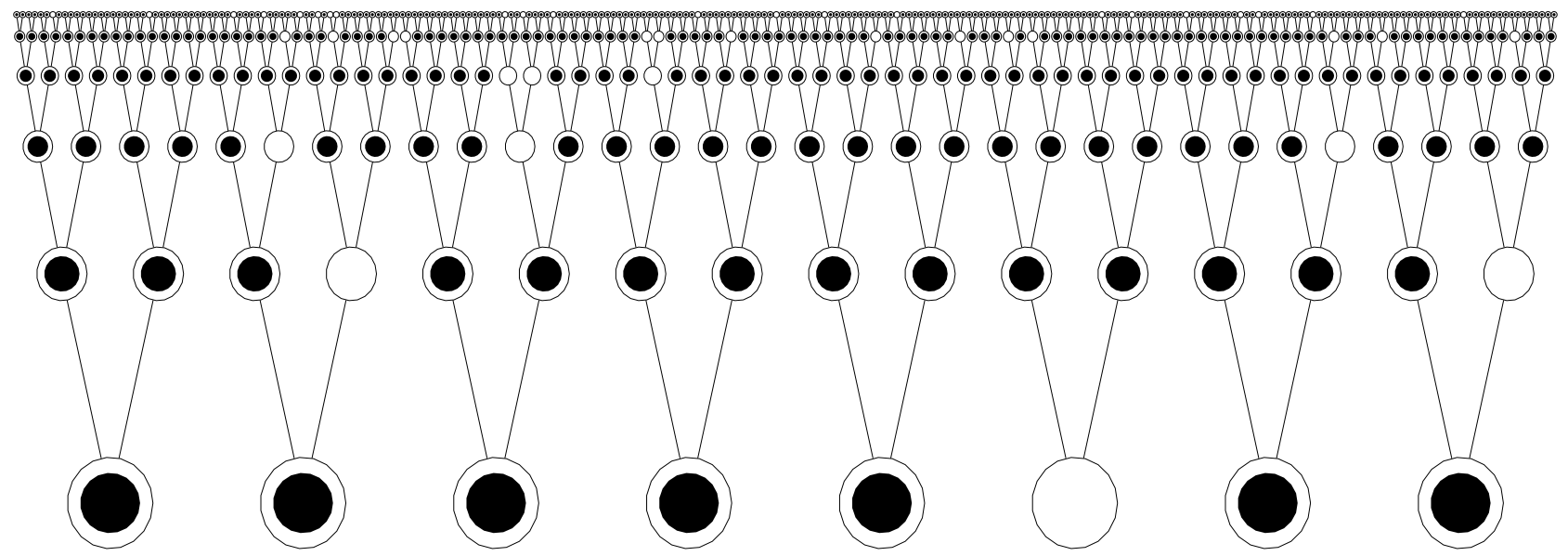

FIG. 1. - Schematic of a binary merger tree for an ejection probability of $p_{\mathrm{ej}}=0.1$ after six generations. The circles represent galaxies while the black dots in their centers symbolize the presence of a SMBH.

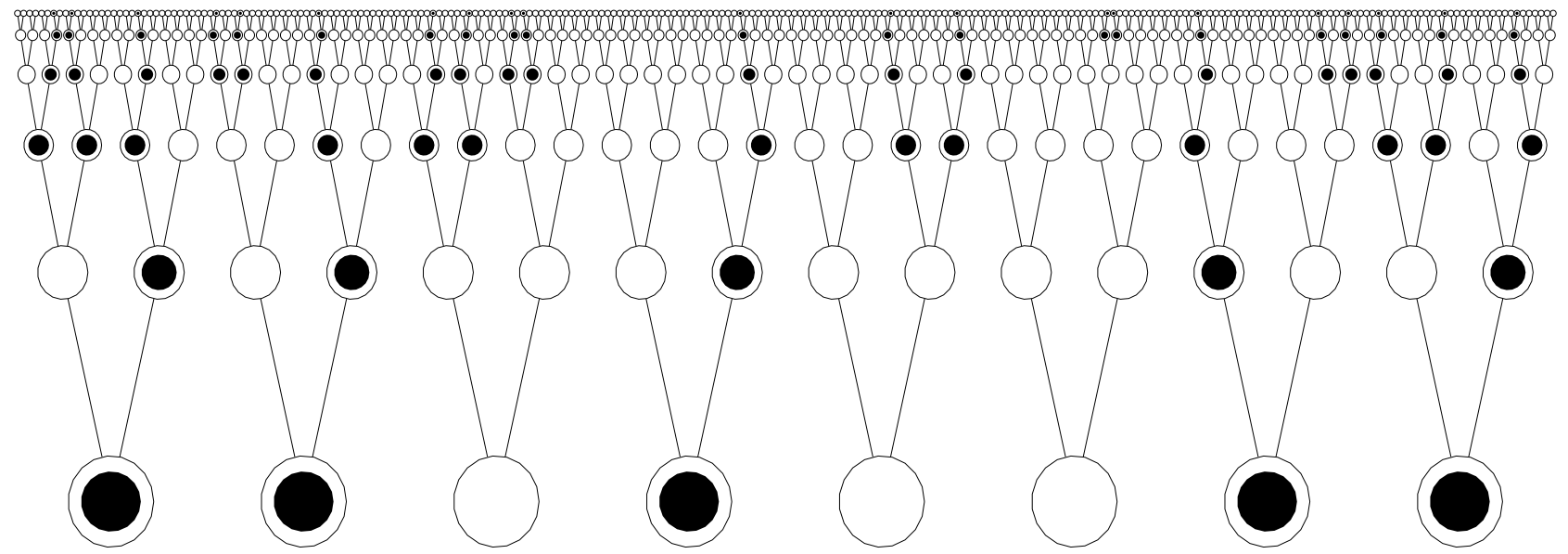

FIG. 2.- Merger tree for an ejection probability of $p_{\text {ej }}=0.9$ after six generations. 

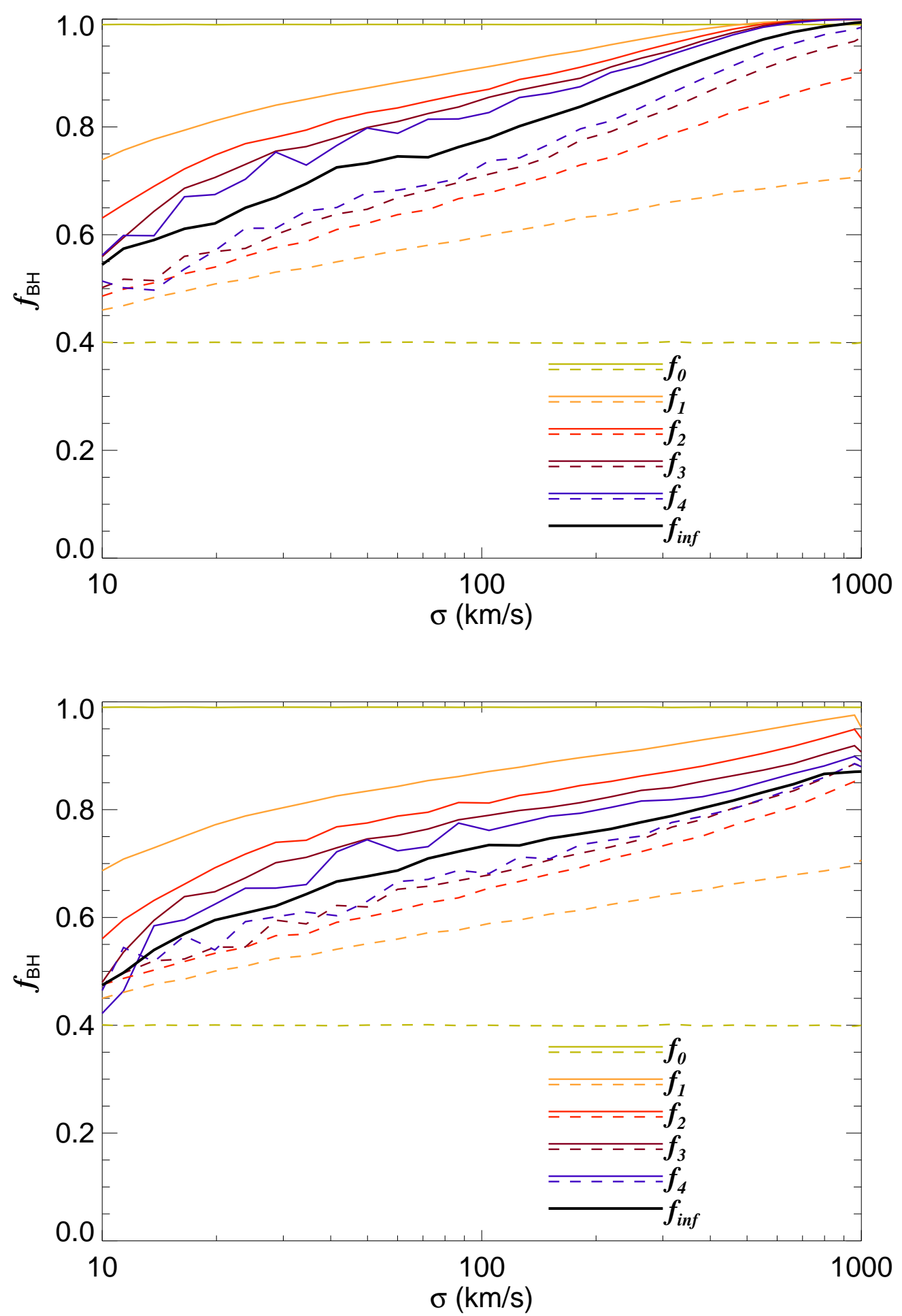

FIG. 3.- BH occupation fraction as a function of the galactic velocity dispersion $\sigma_{v}$ for the Monte Carlo merger model with $v_{\text {esc }}=2 \sigma_{v}$ and the recoil velocity distribution of Schnittman \& Buonanno (2007) (upper panel). The solid curves correspond to an initial occupation fraction $f_{0}=0.99$ and the dashed curves $f_{0}=0.4$, with $f_{i}$ the occupation fraction after an average of $i$ mergers per galaxy. Both models converge to a single value of $f_{\infty}$, shown as a thick black curve. In the lower panel, we show the occupation fraction for an average kick velocity three times as large as that predicted by Schnittman \& Buonanno (2007). In all cases, we set the merger fraction in each generation as $f_{m}=0.66$. 